\title{
MiR-454 Prompts Cell Proliferation of Human Colorectal Cancer Cells by Repressing CYLD Expression
}

\author{
Hong-Liang Liang ${ }^{1,3}$, Ai-Ping $\mathrm{Hu}^{2}$, Sen-Lin $\mathrm{Li}^{3}$, Jia-Ping $\mathrm{Xie}^{3}$, Qing-Zhu Ma ${ }^{3}$, \\ Ji-Yong Liu ${ }^{1 *}$
}

\begin{abstract}
Previous studies have shown that miR-454 plays an important role in a variety of biological processes in various human cancer cells. However, the underlying mechanisms of this microRNA in colorectal cancer (CRC) cells remain largely unknown. In the present study, we investigated the miR-454 role in CRC cell proliferation. We found that miR-454 expression is markedly upregulated in CRC tissues and CRC cells compared with the matched tumor adjacent tissues and the FHC normal colonic cell line. Ectopic expression of miR-454 promoted the proliferation and anchorage-independent growth of CRC cells, whereas inhibition of miR-454 reduced this effect. Bioinformatics analysis further revealed cylindromatosis (CYLD), a putative tumor suppressor as a potential target of miR-454. Data from luciferase reporter assays showed that miR-454 directly binds to the 3'-untranslated region (3'-UTR) of CYLD mRNA and repressed expression at both transcriptional and translational levels. In functional assays, CYLD-silenced in miR-454-in-transfected SW480 cells have positive effect to promote cell proliferation, suggesting that direct CYLD downregulation is required for miR-454-induced CRC cell proliferation. In sum, our data provide compelling evidence that miR-454 functions as an onco-miRNA, playing a crucial role in the promoting cell proliferation in CRC, and its oncogenic effect is mediated chiefly through direct suppression of CYLD expression.
\end{abstract}

Keywords: miR-454 - colorectal cancer - CYLD - cell proliferation

Asian Pac J Cancer Prev, 16 (6), 2397-2402

\section{Introduction}

Colorectal cancer (CRC) is the third most commonly diagnosed carcinoma in males and the second in females (Jemal et al., 2011). Surgery is the main treatment for operable colorectal cancer, followed by chemotherapy and radiation (Aschele et al., 2009; Suppiah et al., 2009; Andre et al., 2010; Primrose, 2010). However, recurrence and metastasis are very common. Thus, there is an urgent need to elucidate the underlying molecular mechanisms of CRC and find new molecular targets for treatment of this disease.

Recently, microRNAs (miRNAs) were discovered to be the endogenous non-coding small RNA (19-22 nucleotides), which lead to mRNA degradation or inhibition of translation through imperfect hybridization to 3'-untranslated region (3'-UTR) in target mRNAs that directly regulate the expression of target genes (Ambros, 2004; Bartel, 2004; Chen et al., 2014). Among several candidate miRs regulating cell proliferation, miR-454 has been shown to be one of the important determinants of cell proliferation in several kinds of cancers. Thereafter, it has shown that miR-454 modulates a lot of target genes, among these genes, cylindromatosis (CYLD) has been shown to be involved in regulating the apoptosis of cancer cells (Massoumi et al., 2006; Miliani de Marval et al., 2011). Cell proliferation, which is the most important hallmarks of cancer, is the leading lethal factors for malignant cancer, especially for CRC (Gao et al., 2013; Raman et al., 2013). However, the relationship between $\mathrm{CRC}$ and the expression of miR-454 has not yet been elucidated.

In the present study, we report that upregulation of miR-454 in CRC is associated with development of CRC. Further investigations revealed that miR-454 directly targeted the 3'-UTR of CYLD to suppress the expression of this gene, which in turn promoted the proliferation of CRCs.

\section{Materials and Methods}

\section{Clinical specimens}

Eight paired human colorectal cancer tissues and the matched tumor adjacent tissues (TAT) were obtained from CRC patients and histopathologically diagnosed at Shandong Provincial Hospital, Shandong University 
(Jinan, People's Republic of China). The study was approved by the ethics committee of Shandong Provincial Hospital, Shandong University (Jinan, People's Republic of China). Written informed consent was obtained from all patients. Tissue samples were collected at surgery, immediately frozen in liquid nitrogen and stored until total RNAs or proteins were extracted.

\section{Cell culture}

Human CRC cell lines SW403, HT-29, COLO320DM, COLO205, SW480, KM202L, SW620 and normal colonic cell line FHC were purchased from National Rodent Laboratory Animal Resource (Shanghai, People's Republic of China). All CRC cell lines were grown in Dulbecco's Modified Eagle Medium (DMEM, Gbico, USA) supplemented with $10 \%$ fetal bovine serum (FBS, Sigma, USA), 100units/ml of penicillin-streptomycin (Invitrogen, Carlsbad, CA), and Normal colon FHC cells were grown in DMEM/F-12 medium with $10 \%$ FBS, 10ng/mL cholera toxin, $5 \mu \mathrm{g} / \mathrm{mL}$ transferrin, $5 \mu \mathrm{g} /$ $\mathrm{mL}$ insulin, $100 \mathrm{ng} / \mathrm{mL}$ hydrocortisone and extra $10 \mathrm{mM}$ 4-(2-hydroxyethyl)-1-piperazineëthanesulfonic acid (HEPES). Cell lines were cultured in a humidified incubator at $37^{\circ} \mathrm{C}$ in an atmosphere of $5 \% \mathrm{CO} 2$ and $95 \%$ air.

\section{Plasmids, small interfering RNA and transfection}

For ectopic expression of CYLD, CYLD ORFs with 3'-UTR was amplified using PCR and subcloned into pEGFP-C1 (Clontech, Mountain View, CA, USA) and pGL3 vectors (Promega, Madison, WI, USA). The primers used (forward and reverse) were as follows: CYLD-3'UTR-GFP-up, 5'-GCCCTCGAGCTTGACTCCGTTCCCCTTCAGAC3'; CYLD-3'UTR-GFP-dn, 5'-GCCGGA T C C A A C C A A G G G C A G T T G A G T C - 3', a $\mathrm{nd} \quad \mathrm{C}$ Y L D - 3, U T R - $1 \mathrm{u} \mathrm{c}-\mathrm{u} \mathrm{p}$, 5'-GCCCCGCGGCTCCGTTCCCCTTCAGAC-3'; CYLD-3'UTR-1uc-dn, 5'-GCCCTGCAGAAC CAAGGGCAGTTGAGTC-3'. For CYLD depletion, small interfering RNA (siRNA) was synthesized and purified by RiboBio Co. (Guangzhou, Guangdong, China). The siRNAs used were CYLD siRNA\#1: 5'-GUACCGAAGGGAAGUAUAGUU-3' and CYLD SiRNA\#2: 5'-CGCGCUGUAACUCUUUAGCAUU-3'. MiR-454 mimic, miR-454 inhibitor and negative control were purchased from RiboBio (Guangzhou, Guangdong, China). Transfection of plasmid and siRNAs were performed using lipofectamine 2000 (Invitrogen), according to the manufacturer's protocol.

\section{RNA extraction and real-time quantitative PCR}

For miRNA quantification, total RNA was extracted from culture cells and patient samples using mirVana miRNA Isolation Kit (Ambion) according to the manufacturer's instructions, and then cDNA was synthesized from $5 \mathrm{ng}$ of total RNA using the Taqman miRNA reverse transcription kit (Applied Biosystems). The expression levels of miR-454 were quantified using the miRNA-specific TaqMan MiRNA Assay Kit (Applied Biosystems). The relative miR-454 expression levels after normalization to U6 small nuclear RNA were calculated using 2-[( $\mathrm{Ct}$ of miR-454)-(Ct of U6)]. Quantitative PCR was performed by SYBR Kit (Qiagen, China) using a Light Cycler system. The primers selected are as follows: Cyclin D1 forward 5'-TCCTCTCCAAAATGC CAGAG-3' and Cyclin D1 reverse 5'-GGCGGATTGGAAATGAACTT-3'; Myc forward 5'-TCAAGAGG CGAACACACAAC-3' and reverse 5'-GGCCTTTTCATTGTTTTCCA-3'. Expression data was normalized to the geometric mean of GAPDH to control the variability in expression levels (forward primer 5'- GACTCATGACCACAGT CCATGC-3'; reverse primer 3'- AGAGGCAGGGATGATGTTCTG-5') and calculated as 2-[(Ct of CyclinD1 and $\mathrm{Myc})-(\mathrm{Ct}$ of GAPDH)].

\section{MTT assays and colony formation}

Cell growth was measured by MTT assay, SW480 cells were seeded in 96-well plates in medium containing $10 \%$ FBS at approximately 2,000 cells/well. For quantitation of cell viability, cultures were stained after 1,2, 3 and 4 days in MTT assays. In brief, $20 \mu \mathrm{l}$ of $5 \mathrm{mg} / \mathrm{ml} \mathrm{MTT}$ solution (Sigma-Aldrich) was added to each well and incubated for $4 \mathrm{~h}$ at $37^{\circ} \mathrm{C}$. After the incubation period, the culture medium was removed and $150 \mu \mathrm{L}$ DMSO (Sigma-Aldrich) was added. The absorbance at $490 \mathrm{~nm}$ was measured in a Thermo Scientific Multiskan (Thermo Fisher Scientific, USA).

For colony formation assay, SW480 cells were plated 6-well plates (1000 cells per well) and incubated for 10 days in medium containing $10 \% \mathrm{FBS}$. Colonies were fixed with $10 \%$ formaldehyde for $5 \mathrm{~min}$ and stained with $1.0 \%$ crystal violet for $30 \mathrm{~s}$. The number of colonies, defined as $>50$ cells/colony were counted. Three independent experiments were performed and the data was calculated using paired t test.

\section{Anchorage-independent growth assay}

Cells were trypsinized, and 1000 cells were resuspended in $2 \mathrm{ml}$ complete medium plus $0.3 \%$ agar (Sigma). The agar-cell mixture was plated on top of a bottom layer consisting of $1 \%$ agar in complete medium. The plates were incubated at $37^{\circ} \mathrm{C}$ in a humid atmosphere of $5 \% \mathrm{CO} 2$. Cells were incubated for 2 weeks at $37^{\circ} \mathrm{C}$ until colony formation and colonies were stained with $0.5 \%$ Crystal Violet for counting under microscope and cell colonies were photographed at an original magnification of $100 \times$. Only cell colonies containing more than 50 cells were counted. The experiment was performed for 3 independent times for each cell line.

\section{Luciferase assays}

Cells were seeded in triplicate in 24-well plates $\left(5 \times 10^{4} /\right.$ well) and cultured for 24 . The pGL3-luciferase reporter gene plasmids pGL3-CYLD-3'-UTR (wt/mut), or the control-luciferase plasmid were cotransfected into the cells with 5ng pRL-TK Renilla plasmid (Promega) using Lipofectamine 2000 Reagent (Invitrogen). Luciferase and Renilla activities were assayed $36 \mathrm{~h}$ after transfection using the dual luciferase assay kit to the manufacturer's protocol. 


\section{Western blotting}

Protein lysates were prepared, equal quantities of protein were s subjected to SDS-PAGE, gels were transferred onto PVDF membranes for two hours at $4^{\circ} \mathrm{C}$, run at a current of $125 \mathrm{~mA}$ and blocked for two hours. The membrane was incubated overnight with anti-CYLD, anti-Cyclin D1 and anti-c-Myc (1:1000; Cell Signaling Technology) and washed with tris buffered saline and Tween-20 (TBST) three times, for five minutes each time. To control sample loading, the blotting membranes were stripped and re-probed with an anti-a-tubulin antibody (Sigma-Aldrich). After being washed with TBST and incubation with either anti-rabbit horseradish peroxidaseconjugated secondary antibody (Sigma-Aldrich) for $2 \mathrm{~h}$ at room temperature, immunocomplexes were visualized using the chemiluminesence (GE, USA) following the manufacturer's protocol.

\section{Statistical analysis}

All statistical analyses except for microarray data were performed using the SPSS 19.0 (IBM). Student's t test was used to evaluate the significance of the differences between two groups of data in all the pertinent experiments. A $p<0.05$ (using a two-tailed paired t-test) was thought to be significantly different for two groups of data.

\section{Results}

MiR-454 expression was upregulated in CRC tissues and CRC cell lines

To investigate the role of miR-454 in CRC development, we evaluated the expression levels of miR-454 in CRC tissues and CRC cells by qRT-PCR. As showed in figure $1 \mathrm{~A}$, the results showed that the expression levels of miR454 were consistently upregulated in the CRC tissues compared with the matched tumor adjacent tissues, and in all 8 tested CRC cell lines had significantly upregulated miR-454 levels than those in the normal colonic cell line FHC. These results showed that miR-454 played an important role in CRC. Together, these results suggest that miR-454 is significantly increased in CRC and may serve as a prognostic marker for patients with CRC. MiR454 expression in SW480 cells was less abundant than in COLO320DM, KM202L and SW620, and more abundant than in SW403, HT-29 and COLO205. Thus, SW480 cells appeared as the fitted cellular models for processing up and down regulation of $\mathrm{miR}-454$.

\section{MiR-454 promoted $C R C$ cell proliferation}

To investigate whether CRC cell proliferation was regulated by miR-454, we investigated whether miR-454 plays a tumor positive role in cell proliferation of CRC. We transfected the SW480 cells with miR-454 mimics, miR454 inhibitor or the respective controls. Relative miR-454 expression was verified using qRT-PCR (Figure 2A and $3 \mathrm{~A})$. We first evaluated the effect of miR-454 on cell proliferation using MTT, colony formation and anchorageindependent growth, respectively. To investigate the role of miR-454 upregulation in the development and progression of CRC, we next examined its effect on cellular proliferation. MTT assay showed that miR-454
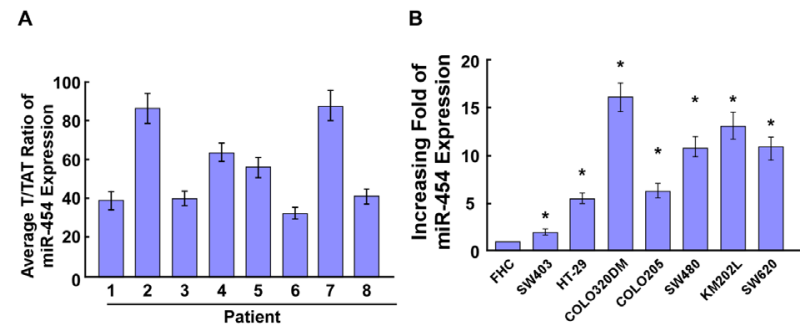

Figure 1. Expression of miR-454 in Human Colorectal Cancer (CRC) Tissues and Cell Lines. (A) Relative miR-454 mRNA expression levels in 8 paired primary CRC tissues $(\mathrm{T})$ and the matched tumor adjacent tissues (TAT) from the same patient were detected by PCR analysis. (B) Real-time PCR analysis of miR-454 expression in FHC cells and CRC cell lines, including SW403, HT-29, COLO320DM, COLO205, SW480, KM202L and SW620. Experiments were repeated at least three times (A and B). Each bar represents the mean of three independent experiments. ${ }^{*} p<0.05$
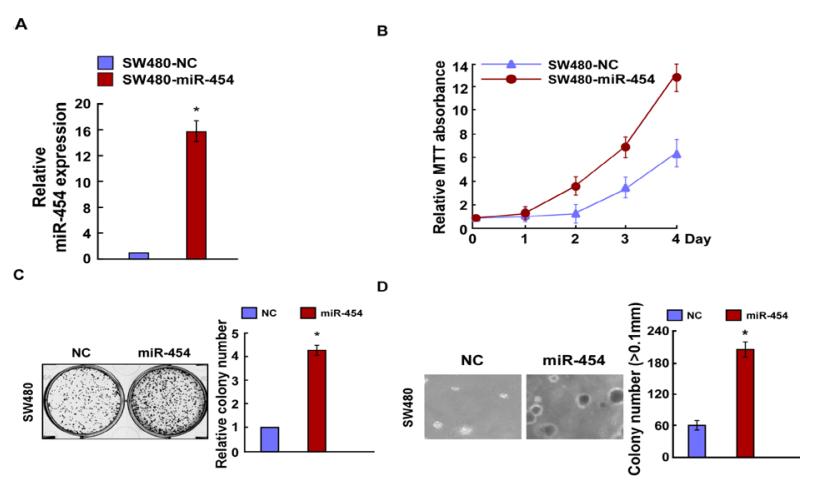

Figure 2. MiR-454 Upregulation Promoted CRC Cell Proliferation. (A) Validation of miR-454 expression levels after transfection by PCR analysis. (B) MTT assays revealed that upregulation of miR-454 promoted growth of SW480 cells. (C) Representative micrographs (left) and quantification (right) of crystal violet-stained cell colonies. (D) Upregulation of miR-454 promoted the anchorage-independent growth of SW480 cells. Representative micrographs (left) and quantification of colonies that were $>0.1 \mathrm{~mm}$ (right). Each bar represents the mean of three independent experiments. ${ }^{*} p<0.05$

upregulation significantly increased the proliferation rate of SW480 cells (Figure 2B), and this was further confirmed by a colony formation assay (Figure 2C). Strikingly, we found that enforced expression of miR-454 in SW480 cells drastically enhanced their anchorageindependent growth ability (Figure 2D). In contrast, the cell growth rates and colony numbers of SW480 cells transfected with miR-454 inhibition (miR-454-in) were significantly inhibited the cell growth rate than those transfected with NC (Figure 3B and C). In addition, the anchorage-independent growth ability of SW480 cells was significantly decreased in response to miR-454-in (Figure 3D). These results showed that miR-454 increased CRC cell tumorigenicity in vitro.

MiR-454 directly targets CYLD by binding to its 3'-UTR

It is generally accepted that miRNAs exert their functions by regulating their downstream target genes expression. Potential targets of miR-454 were predicted 


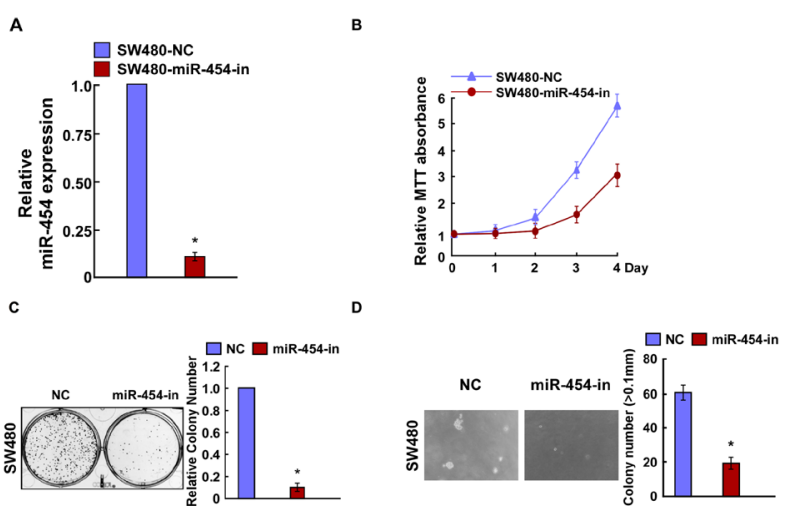

Figure 3. Inhibition of miR-454 Inhibited CRC Cell Proliferation. (A) Validation of miR-454 expression levels after transfection by PCR analysis. (B) MTT assays revealed that inhibition of miR-454 suppressed growth of SW480 cells. (C) Representative micrographs (left) and quantification (right) of crystal violet-stained cell colonies. (D) Inhibition of miR-454 inhibited the anchorage-independent growth of SW480 cells. Representative micrographs (left) and quantification of colonies that were $>0.1 \mathrm{~mm}$ (right). Each bar represents the mean of three independent experiments. ${ }^{*} p<0.05$

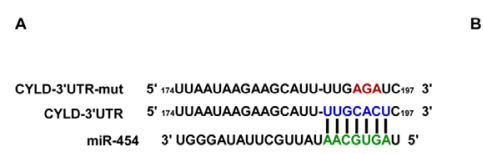

C
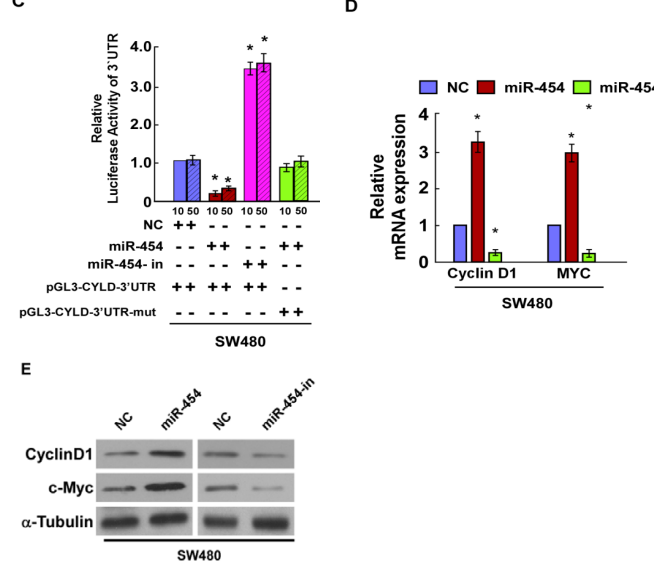

Figure 4. MiR-454 Suppresses CYLD Expression By Directly Targeting the CYLD $3^{\prime}$-UTR and Altered Levels of Proteins Related to Proliferation or Apoptosis in SW480 Cells. (A) Predicted miR-454 target sequence in the 3'-UTR of CYLD (CYLD-3'-UTR) and positions of three mutated nucleotides (red) in the 3'-UTR of CYLD (CYLD3'-UTR -mut). (B) CYLD protein expression in SW480 cells transfected with miR-454 or the miR-454 inhibitor were detected by Western blotting analysis. $\alpha$-Tubulin served as the loading control. (C) Luciferase reporter assay of SW480 cells transfected with the pGL3-CYLD-3'-UTR reporter or pGL3-CYLD-3'UTR-mut reporter and miR-454 or miR-454-in with increasing amounts (10 and $50 \mathrm{nM}$ ) oligonucleotides. (D) Real-time PCR analysis of expression of Cyclin D1 and MYC in SW480 cells. (E) Western blotting analysis of protein expression of Cyclin D1 and c-Myc in SW480 cells. $\alpha$-Tubulin was used to serve as the loading control. ${ }^{*} p<0.05$ using bioinformatics methods. CYLD, a tumor suppressor gene containing a binding site for miR-454, was selected as the target for further analysis (Figure 4A).

To determine whether miR-454 affects CYLD expression, expression of CYLD were detected in the SW480 cells, which were transfected with miR-454 mimics, miR-454-in or the respective controls. Western blotting analysis showed that miR-454 mimics markedly suppressed CYLD protein levels in SW480 cells (Figure 4B), while miR-454-in clearly promoted CYLD protein expression. To verify the effect of miR-454 on the inhibition of CYLD expression, we examined whether CYLD is regulated by miR-454 through direct binding to its 3'UTR. CYLD 3'-UTR (wild or mut) vector were cotransfected in SW480 cells with miR-454mimic, miR454-in or miR-NC, followed by measurement of luciferase activity. As shown in figure 4C, a consistent and dosedependent reduction of luciferase activity was observed in SW480 cells transfected with miR-454 mimic, whereas the repressive effect of miR-454-in increased wild-type CYLD luciferase activity. Meanwhile, overexpressing miR-454 had no effect on the luciferase activity of CYLD 3'-UTR mut type. These results, taken together, demonstrated that CYLD is a bona fide target of miR-454.

\section{MiR-454 altered levels of proteins related to proliferation in CRC cells}

We had resulted that miR-454 could promote the growth and proliferation of CRCs and CYLD was a direct target of miR-454. It was reported previously that CYLD is closely correlated with cell proliferation. Meanwhile, we observed that the mRNA of the CYLD downstream gene, Cyclin D1 and c-Myc, which is a number of critical cellproliferation regulators. c-Myc is a nuclear transcriptional factor and most of its targeting genes including Cyclin D1 are crucial in the regulation of cell proliferation, differentiation, apoptosis and other cellular functions (Roy et al., 1993). Furthermore, the expression of Cyclin D1

A

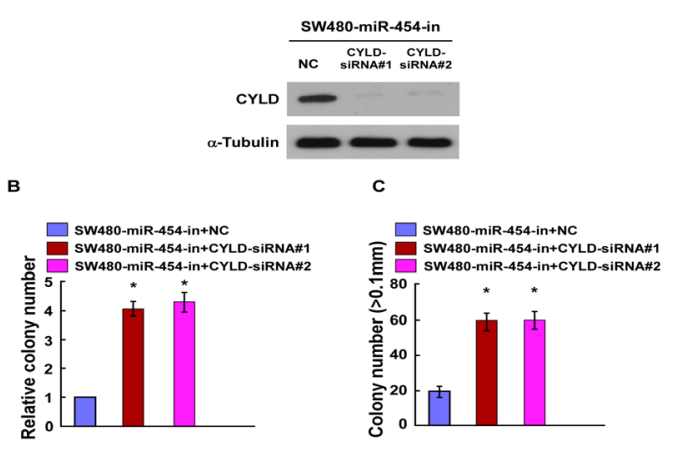

Figure 5. CYLD downregulation is required for miR454-induced proliferation of CRC cells. (A) Western blot analysis verified that silencing CYLD effectively decreased the expression of CYLD in miR-454-in-transfected SW480 cells. (B) miR-454-in-transfected SW480 cells after transfection with CYLD-siRNAs have an additive effect to promote the anchorageindependent growth. (C) Representative quantification of colonies that were $>0.1 \mathrm{~mm}$. Each bar represents the mean of three independent experiments. $* p<0.05$ 
and c-Myc were upregulated in SW480 cells transfected with miR-454 mimic, but decreased in the cells transfected with miR-454-in, relative to control cells (Figure $4 \mathrm{E}$ ). Thus providing further evidence that miR-454 plays an important role in CRC cell proliferation. Altogether, our results indicated that miR-454 functionally modulates cellular proliferation regulators, Cyclin D1 and C-Myc, thus relevant to cell proliferation.

CYLD downregulation is required for miR-454 -induced proliferation of CRC cells

To further investigate the role of CYLD reduction in miR-454-induced CRC proliferation, we first examined the effects of CYLD downregulation on CRC cell proliferation. As predicted, Western blot analysis verified that CYLD-siRNA\#1 and CYLD-siRNA\#2 both effectively decreased the expression of CYLD in miR-454in-transfected SW480 cells (Figure 5A). Colony formation and anchorage-independent growth assays showed that CYLD-silenced in miR-454-in-transfected SW480 cells have an additive effect on cells proliferation (Figure 5 $\mathrm{B}$ and $\mathrm{C}$ ). Taken together, these results demonstrate that direct CYLD downregulation is required for miR-454induced CRC cell proliferation.

\section{Discussion}

It has been well established that microRNAs (miRNAs) are a large group of post-transcriptional gene regulators that potentially play essential roles in multiple biological processes, including cell differentiation, proliferation, angiogenesis, invasion and migration (Bartel, 2004; Esquela-Kerscher and Slack, 2006; Shi et al., 2014; Zhong et al., 2014). Recently, deregulation of miRNAs in cancer cells and their roles in tumorigenesis have been increasingly investigated (Calin and Croce, 2006; Esquela-Kerscher and Slack, 2006; Rico-Rosillo et al., 2014). However, it was uncertain whether dysregulation of miR-454 was associated with the progression of CRC.

In this study, we found that miR-454 expression was markedly upregulated in CRC tissues and CRC cells. Furthermore, ectopic expression of miR-454 enhanced the proliferation and anchorage-independent growth of CRC cells, whereas miR-454-in had the opposite effect. Bioinformatic algorithms predicted that cylindromatosis (CYLD), which was bona fide target genes of miR-454. CYLD gene is involved in tumor progression by acting as a negative regulator of nuclear factor $-\varkappa \mathrm{B}(\mathrm{NF}-\varkappa \mathrm{B})(\mathrm{Xia}$ et al., 2014; Zhang et al., 2014). Iliopoulos et al. (2010) indicated that miR-181b-1 targets CYLD, leading to increased NF- $x \mathrm{~B}$ activity, which is required to maintain the transformed state in ER-Src cells. CYLD has been recognized as a tumor suppressor due to its dominant genetic linkage to multiple types of cancers (Miliani de Marval et al., 2011; Kinoshita et al., 2013). More specifically, it was reported previously that CYLD is closely correlated with cell proliferation (Alameda et al., 2010). The expression levels of a number of critical cell-proliferation regulators were also detected. Among cell proliferation related genes, we focus on c-Myc gene, which is a very strong proto-oncogene, is often found to be up-regulated in many types of cancers including CRC. c-Myc is a nuclear transcriptional factor and most of its targeting genes including Cyclin D1 are crucial in the regulation of cell proliferation (Roy et al., 1993; Ratschiller et al., 2003; Knudsen et al., 2006; Sharma et al., 2013). In this study, we showed that the molecular mechanism by which miR-454 promotes CRC cell proliferation was due to upregulation of cyclin D1 and c-MYC via repressing CYLD in cells overexpressing miR-454, which are two miR-454 targeted proteins that positively control cell proliferation. Furthermore, CYLDsilenced in miR-454-in-transfected SW480 cells have positive effect to promote cell proliferation, suggesting that direct CYLD downregulation is required for miR454-induced CRC cell proliferation.

In conclusion, the current study revealed that miR-454 is a proto-oncogenic miRNA in CRC cells and that CYLD is a novel and critical miRNA-454 target. Therefore, all the results indicated that miR-454 might serve as a potential therapeutic marker for treating CRC.

\section{Acknowledgements}

This work was supported by Department of Gastroenterology, Shandong Provincial Hospital.

\section{References}

Alameda JP, Moreno-Maldonado R, Navarro M, et al (2010). An inactivating CYLD mutation promotes skin tumor progression by conferring enhanced proliferative, survival and angiogenic properties to epidermal cancer cells. Oncogene, 29, 6522-32.

Ambros V (2004). The functions of animal microRNAs. Nature, 431, 350-5.

Andre T, Tuvignon N, Taieb J, et al (2010). Operable colon cancer: initial strategy. Rev Prat, 60, 1089-93.

Aschele C, Bergamo F, Lonardi S (2009). Chemotherapy for operable and advanced colorectal cancer. Cancer Treat Rev, 35, 509-16.

Bartel DP (2004). MicroRNAs: genomics, biogenesis, mechanism, and function. Cell, 116, 281-97.

Calin GA, Croce CM (2006). MicroRNA signatures in human cancers. Nat Rev Cancer, 6, 857-66.

Chen P, Wang BL, Pan BS, et al (2014). MiR-1297 regulates the growth, migration and invasion of colorectal cancer cells by targeting cyclo-oxygenase-2. Asian Pac J Cancer Prev, 15, 9185-90.

Esquela-Kerscher A, Slack FJ (2006). Oncomirs-microRNAs with a role in cancer. Nat Rev Cancer, 6, 259-69.

Gao Y,Li BD, Liu YG (2013). Effect of miR27a on proliferation and invasion in colonic cancer cells. Asian Pac J Cancer Prev, 14, 4675-8.

Iliopoulos D, Jaeger SA, Hirsch HA, et al (2010). STAT3 activation of miR-21 and miR-181b-1 via PTEN and CYLD are part of the epigenetic switch linking inflammation to cancer. Mol Cell, 39, 493-506.

Jemal A, Bray F, Center MM, et al (2011). Global cancer statistics. CA Cancer J Clin, 61, 69-90.

Kinoshita H, Okabe H, Beppu T, et al (2013). CYLD downregulation is correlated with tumor development in patients with hepatocellular carcinoma. Mol Clin Oncol, 1, 309-14.

Knudsen KE, Diehl JA, Haiman CA, et al (2006). Cyclin D1: 
polymorphism, aberrant splicing and cancer risk. Oncogene, 25, 1620-8.

Massoumi R, Chmielarska K, Hennecke K, et al (2006). Cyld inhibits tumor cell proliferation by blocking Bcl-3-dependent NF-kappaB signaling. Cell, 125, 665-77.

Miliani de Marval P, Lutfeali S, Jin JY, et al (2011). CYLD inhibits tumorigenesis and metastasis by blocking JNK/ AP1 signaling at multiple levels. Cancer Prev Res, 4, 851-9.

Primrose JN (2010). Surgery for colorectal liver metastases. $B r$ J Cancer, 102, 1313-8.

Raman M, Ambalam P, Kondepudi KK, et al (2013). Potential of probiotics, prebiotics and synbiotics for management of colorectal cancer. Gut Microbes, 4, 181-92.

Ratschiller D, Heighway J, Gugger M, et al (2003). Cyclin D1 overexpression in bronchial epithelia of patients with lung cancer is associated with smoking and predicts survival. $J$ Clin Oncol, 21, 2085-93.

Rico-Rosillo MG, Vega-Robledo GB, Oliva-Rico D (2014). The role and importance of the microRNAs in the diagnosis and development of diseases. Rev Med Inst Mex Seguro Soc, 52, 302-7.

Roy AL, Carruthers C, Gutjahr T, et al (1993). Direct role for Myc in transcription initiation mediated by interactions with TFII-I. Nature, 365, 359-61.

Sharma M, Sharma S, Arora M, et al (2013). Regulation of cellular Cyclin D1 gene by arsenic is mediated through miR-2909. Gene, 522, 60-4.

Shi L, Wang Z, Sun G, et al (2014). miR-145 inhibits migration and invasion of glioma stem cells by targeting ABCG2. Neuromolecular Med, 16, 517-28.

Suppiah A, Hartley JE, Monson JR (2009). Advances in radiotherapy in operable rectal cancer. Dig Surg, 26, 187-99.

Xia JT, Chen LZ, Jian WH, et al (2014). MicroRNA-362 induces cell proliferation and apoptosis resistance in gastric cancer by activation of NF-kappaB signaling. J Transl Med,12,33.

Zhang L, Dong Y, Zhu N, et al (2014). microRNA-139-5p exerts tumor suppressor function by targeting NOTCH1 in colorectal cancer. Mol Cancer, 13, 124.

Zhong Q, Wang T, Lu P, et al (2014). miR-193b promotes cell proliferation by targeting Smad3 in human glioma. $J$ Neurosci Res, 92, 619-26. 